\title{
Search for a diffuse flux of cosmic neutrinos with the ANTARES neutrino telescope
}

\section{L.A. Fusco ${ }^{a, *}$ on behalf of the ANTARES Collaboration}

(a complete list of authors can be found at the end of the proceedings)

${ }^{a}$ Aix Marseille Univ, CNRS/IN2P3, CPPM, Marseille, France

E-mail: fusco@cppm.in2p3.fr

The previous analysis of the ANTARES all-flavour 12-year neutrino data sample provided the observation of an excess of events, at the highest energies, above the expected atmospheric foregrounds. This excess, even though mild $(1.8 \sigma)$, has been found to be consistent in spectral slope and normalisation with the high-energy diffuse cosmic neutrino signal detected by the IceCube Neutrino Observatory. Even though the smaller detector size does not provide sufficient statistics to claim an independent discovery, the analysis of ANTARES data can provide valuable information in the study of the high-energy neutrino signal, in particular for what concerns the details of its energy distribution in the case of soft-spectra solutions. To improve the previous ANTARES results, a new event selection has been developed for cascade-like events, relying on a Boosted Decision Tree multivariate-analysis technique. This increased the event statistics in this channel by a factor of 5 , while also dramatically reducing the surviving foregrounds and the related systematic uncertainties. This contribution will report on the status of the analysis and the prospects emerging from the use of this new event sample in the search for a diffuse flux of cosmic neutrinos.

$37^{\text {th }}$ International Cosmic Ray Conference (ICRC 2021)

July 12 th $-23 r d, 2021$

Online - Berlin, Germany

\footnotetext{
*Presenter
} 


\section{Introduction}

The ANTARES neutrino telescope, a large-volume Cherenkov detector located under the Mediterranean Sea, 40-km off-shore Toulon, France, aims at detecting cosmic neutrinos of any origin. High-energy cosmic neutrinos are believed to be produced in the decay chains of the hadrons and leptons induced by the primary cosmic ray interactions within or around their sources, or as they travel through the Universe. The ensemble of unresolved individual sources that are too faint to be detected, cosmic ray propagation effects, or both, can generate a diffuse flux of cosmic neutrinos.

Such a flux has been detected, at high significance levels, in multiple analyses of data collected by the IceCube Neutrino Observatory. Under the hypothesis of equipartition between the three neutrino flavours as expected in standard production and propagation scenarios, and under the assumption of an unbroken power-law ( $\mathrm{d} \Phi / \mathrm{dE} \propto \mathrm{E}^{-\Gamma}$ ) describing its energy spectrum, this signal is, so far, compatible with the hypothesis of isotropy over the whole sky. The four main analyses that have been carried out by the IceCube Collaboration are: the High Energy Starting Events (HESE) search [1], the search for through-going muons from the Northern Sky [2], the analysis of cascade events [3] and the all-sky combined analysis [4].

Some mild tension is present between the results obtained by the different IceCube analyses. The HESE search reports the observation of a rather soft energy spectrum $(\Gamma \simeq 2.9 \pm 0.2)$ as best-fit option for the cosmic signal. The analysis of upward-going muons from the Northern Sky finds the signal to be compatible with a hard energy spectrum $(\Gamma \simeq 2.3 \pm 0.1)$. The cascade search falls somewhat in between the two, with a best-fit $\Gamma \simeq 2.5 \pm 0.2$. The HESE cosmic signal is statistically dominated by cascade-like events at energies between 50 and $200 \mathrm{TeV}$, mostly coming from the Southern sky. The upwarg-going muons sample in IceCube is most sensitive above a few hundreds of $\mathrm{TeV}$, and only contains events coming from the Northern sky. The difference in the energy range of the different analyses comes from the different atmospheric backgrounds of the two samples, being the atmospheric background in the cascade sample - i.e. a sample dominated by $v_{e}$ interactions - lower in normalisation than the one for upward-going events induced by $v_{\mu} \mathrm{CC}$ interactions.

Multiple-component hypotheses can explain this tension [5]. It has been argued that the Southern sky features the presence of the inner region of the Galactic plane, where soft-spectra neutrino sources could be present, both as individual emitters (mainly Supernovae Remnants) and as the diffuse emission region induced by cosmic rays propagating in the dense interstellar medium in the inner Galactic plane. On the contrary, hard-spectra sources (such as blazars [6]) are expected in the extra-galactic fields, which dominate the Northern hemisphere. The contribution of the inner Galactic Plane diffuse neutrino emission to the all-sky IceCube neutrino signal has been severely constrained by an ANTARES-IceCube joint search [7].

A first hint of a cosmic diffuse flux signal in ANTARES has been spotted in the analysis of 2450 days of high-quality ANTARES data-taking time collected between 2007 and 2015 [8]. That analysis had been updated adding to the data sample 880 days of data-taking time acquired by ANTARES between January 2016 and June 2018, as presented in [9]. Both analyses included track-like and cascade-like events, with the tracks analysis being dominant in statistics, due to the larger effective area, and thus more sensitive to the flux normalisation, and the cascades being 
more sensitive to the energy slope thanks to the better energy resolution that is given by contained cascade-like events.

In this contribution, the viability of further upgrades of the ANTARES diffuse analyses are discussed. In particular, a new event selection chain developed for the measurement of the atmospheric neutrino energy spectrum [10], largely boosting the event statistics in the cascade channel, can be exploited in particular thanks to its higher purity with the respect to the previous samples.

\section{ANTARES data samples}

The ANTARES neutrino telescope has been collecting data with high duty cycle since 2007 . Most of the events that are detected by the detector are downward-going atmospheric muons produced by the primary cosmic ray interaction in the atmosphere. These muons can penetrate to the large depths where the detector is located and produce Cherenkov light that is detected by the PMTs in ANTARES. Neutrinos coming from the same cosmic ray air showers (the so-called atmospheric neutrinos), as well as, possibly, cosmic neutrinos, are much less frequent, more or less by a factor of $10^{5}$ and $10^{7}$ respectively. The so-called track-like neutrino events are induced by charged current weak interactions of muon neutrinos, while cascade-like events are given by all-flavour neutrinos, interacting via all weak interactions channels.

All data collected by the detector are sent to shore where triggering takes place. These events are then reconstructed using maximum-likelihood algorithms that account for the expected amount of light produced by either a muon passing through the detector or by a particle cascade within a fiducial volume around the instrumented volume.

A large quantity of downward-going atmospheric muons is removed by selecting events coming from below the detector, since only neutrinos are able to traverse the Earth. Track-like events, thanks to the long path travelled by the muon, allow for a larger effective surface of the detector since also events produced kilometers away from the apparatus can be detected. The limited size of the cascade-like events produces a signal that can be properly reconstructed only within a certain volume, thus reducing the detection efficiency; however, for the same size-limitations, a more precise measurement of the event energy can be obtained. Both are commonly used in ANTARES analyses to search for both point-source [11] and diffuse flux analyses.

In the case of track-like events, the event selection procedure mainly aims at removing wronglyreconstructed atmospheric muons that end-up in the upward-going sample. This is quite easily accomplished by selecting on the quality parameter of the likelihood reconstruction and the estimated angular error from the same algorithm. This allows to obtain approximately an event rate of one atmospheric neutrino per day with a negligible contamination from wrongly reconstructed atmospheric muons.

Cascade-like events are trickier, since atmospheric muons with catastrophic energy losses along their path directly mimic these events since also in that case a cascade of charged particles is produced. In order to remove these events, a specialised strategy has been developed in [12]. However, the amount of events yielded by this approach was limited $(\sim 0.6$ atmospheric neutrinos per day) and a pretty large fraction of atmospheric muons were still surviving it (20-40\% depending on the energy). 


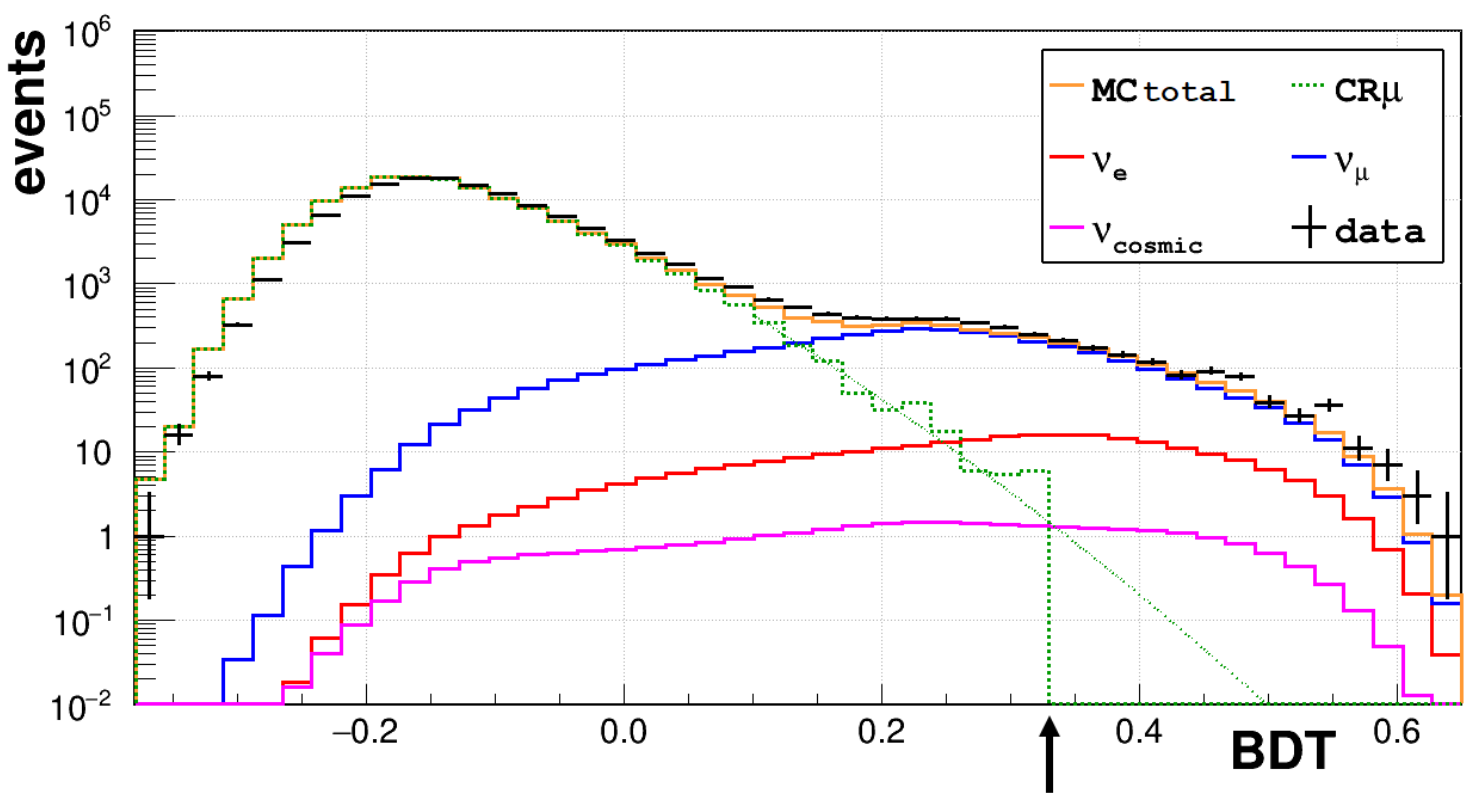

Figure 1: Distribution of the BDT score for data (black crosses) and Monte Carlo (lines) in the 2007-2017 ANTARES sample. Muon and electron atmospheric neutrinos are shown in blue and red respectively. A diffuse cosmic neutrino flux with the spectral shape previously measured in ANTARES [9] is reported in magenta. Atmospheric muons are reported as a dashed green line, together with an extrapolation of their distribution used to evaluate the residual contamination. The orange line shows the sum of all Monte Carlo contributions, and a good data/Monte Carlo agreement is visible in most of the distribution, in particular in the neutrino-dominated part. The arrow shows the BDT value at which the selection cut has been applied for the atmospheric neutrino analysis, which provides a sub-percent contamination from atmospheric muon backgrounds [10].

In order to boost the results coming from this analysis channel, a new selection has been developed. This new approach takes advantage of multivariate analysis techniques (namely a Boosted Decision Tree algorithm) to reject more efficiently atmospheric muon events from data while producing a larger sample of neutrinos passing the selection. Indeed, an event rate of about 0.3 atmospheric neutrino events per day is obtained, with a negligible residual contamination of atmospheric muons. This sample has been used for the first time in the recent ANTARES measurement of the atmospheric neutrino flux [10]. The BDT score distribution is shown in figure 1 .

\section{Binned and unbinned searches}

Previous ANTARES results were based on one-dimensional binned methods. The event selection was optimised on the basis of a model rejection factor procedure [13] so that the best sensitivity to a certain reference cosmic diffuse flux could be achieved. To do so, assumptions were made on the atmospheric foregrounds, with the atmospheric neutrino flux described by the Honda [14] and the Enberg [15] fluxes. The atmospheric muon contribution, simulated in ANTARES with the MUPAGE software [16] was also considered. Once a pre-selection that 
strongly reduced the amount of foreground events was applied, the reconstructed energy estimators of track-like and cascade-like events were used to obtain the best event selection.

The counting analyses yielded a total of 50 ( 27 tracks and 23 cascade) events observed in data in 3300 days of effective livetime, with an expected atmospheric foreground of $36.1 \pm 8.7$ (stat. + syst.) from Monte Carlo simulations (of which 19.9 tracks and 16.2 showers).

The distribution of events surviving the pre-selection had been fitted by means of a binned maximum likelihood method, as previously done in [4] and [8]. Using Monte Carlo simulations, templates of the cosmic signal and of the atmospheric foreground are built. The final likelihood $\mathcal{L}$ is given by the product of the individual likelihoods $\mathcal{L}_{i, S}$ computed for each bin $i$ of the energy estimator distribution for the cascade (c) and track ( $t r)$ samples $S$ separately. The distribution of data and templates are compared considering Poisson statistics, with a Gaussian penalty factor to account for systematic effects on the Monte Carlo input parameters $\tau_{j}^{*}$ :

$$
\begin{aligned}
\mathcal{L} & =\prod_{S \in\{c, t r\}} \prod_{i=0}^{N_{S}} \mathcal{L}_{i, S} \\
\mathcal{L}_{i, S} & =e^{-\mu_{i, S}} \cdot \frac{\mu_{i}^{k_{i, S}}}{k_{i, S} !} \cdot \prod_{j} \frac{1}{2 \pi \sigma\left[\tau_{i, S, j}\right]} e^{-\frac{\left(\tau_{i, S, j}-\tau_{i, S, j}^{*}\right)^{2}}{\sigma^{2}\left[\tau_{i, S, j}\right]}}
\end{aligned}
$$

where $\mu_{i}$ is the expected number of events in the $i$-th bin from the simulated templates, $N_{S}$ is the number of bins in the energy estimator histogram for each event sample and $k_{i, S}$ is the number of events observed in data for that event sample in that bin. The resulting best-fit parameters for the per-flavour cosmic flux normalisation and spectral index are

$$
\begin{aligned}
\Phi_{0}(100 \mathrm{TeV}) & =(1.5 \pm 1.0) \times 10^{-18}\left[\mathrm{GeV}^{-1} \mathrm{~cm}^{-2} \mathrm{~s}^{-1} \mathrm{sr}^{-1}\right] \\
\Gamma & =2.3_{-0.4}^{+0.4}
\end{aligned}
$$

The significance of this excess over the background-only hypothesis has been estimated as of $1.8 \sigma$, by means of a likelihood-ratio test between the best-fit point and the null-cosmic flux outcome.

In order to improve the results of the analysis, apart from moving to the new event selection that provide a larger and more pure event sample in the cascade channel, an unbinned maximum likelihood approach is investigated. A test statistic $\mathcal{T} \mathcal{S}$ is built as:

$$
\mathcal{T S}=\log \mathcal{L}_{\text {sig }}+\text { bkg }-\log \mathcal{L}_{\text {bkg }}
$$

The likelihood function $\mathcal{L}_{\text {sig }+ \text { bkg }}$ is defined as follows:

$$
\mathcal{L}_{\mathrm{sig}+\mathrm{bkg}}=\prod_{\tau \in\{t r, s h\}} \prod_{i \in \tau}\left[\mu_{\mathrm{sig}}^{\tau} \cdot p d f_{\mathrm{sig}}^{\tau}\left(E_{i}, \theta_{i}\right)+\mu_{\mathrm{bkg}}^{\tau} \cdot p d f_{\mathrm{bkg}}^{\tau}\left(E_{i}, \theta_{i}\right)\right]
$$

where product sequences are over the event topology $\tau$ (track-like and shower-like neutrino events) and over each event $i$ belonging to the sample $\tau$. The $E_{i}$ is the reconstructed energy while $\theta_{i}$ is the reconstructed zenith of the event. The $\mu_{\text {sig }}^{\tau}$ parameter is the number of signal events which maximizes the likelihood function while $\mu_{\mathrm{bkg}}^{\tau}$ is the number of background events in the sample.

The signal and background probability density functions (PDFs), $p d f_{\text {sig }}^{\tau}$ and $p d f_{\mathrm{bkg}}^{\tau}$, are built from the Monte Carlo after the selection cuts defined for the track-like and cascade-like samples. 

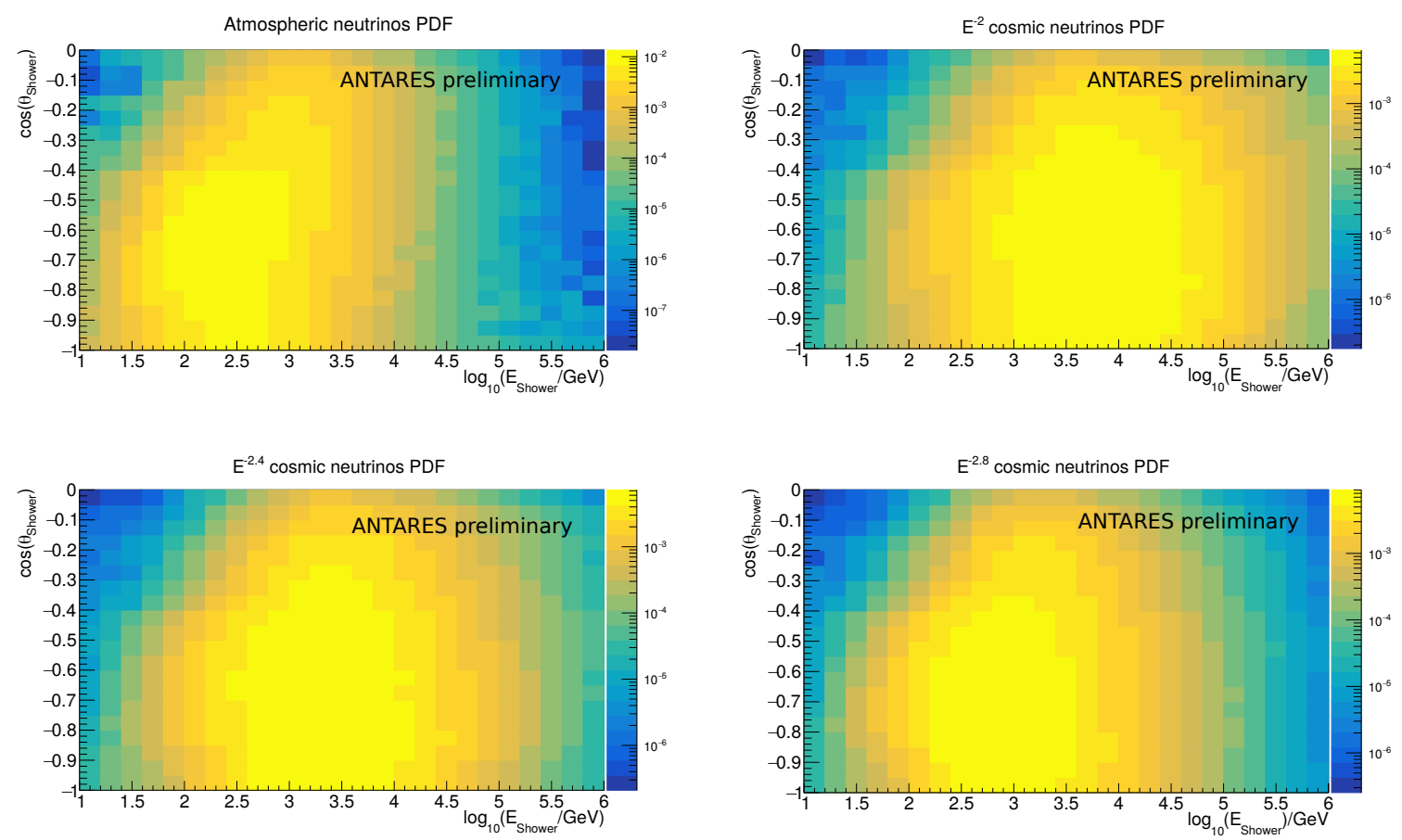

Figure 2: Probability density functions in the cascade channel for atmospheric neutrinos (top left) and cosmic neutrinos for spectral indices $\Gamma=2.0$ (top right), 2.4 (bottom left), 2.8 (bottom right).

The atmospheric neutrino models mentioned above are used for the background term, while the signal flux is taken as an unbroken power-law with spectral index $\Gamma$, which is varied from 1.5 to 3.0 in steps of 0.1. The cascade channel PDFs are shown in figure 2.

The test statistic $\mathcal{T S}$ distributions are then built by means of pseudo-experiments (PE). A total of $5 \cdot 10^{3}$ pseudo-experiments are performed for each PE, in which the search method is applied to pseudo data-sets varying the number of signal events $\mu_{\text {sig }}^{\tau}$ injected in the neutrino sample, from 0 up to 300 in steps of 1 event.

The obtained distributions of $\mathcal{T S}$ for varying $\mu_{\text {sig }}^{\tau}$ are then used to estimate the median sensitivity flux at $90 \%$ confidence level (c.l.) and discovery potential flux at $3 \sigma$ of the pseudo-data sets by comparing them to the background-only $\mathcal{T S}$ distribution.

At this stage, detector systematics are not included in the evaluation, but all normalisation factors for both signal and background are left free in the likelihood fitting. As shown in [8] and [9] the main source of systematic uncertainties in ANTARES was the limited knowledge of the surviving atmospheric muon component in the fit. With the new shower sample, this is completely removed since the sample is so pure in neutrinos that atmospheric muons can be safely neglected.

\section{Results and outlook}

Applying the unbinned method on the pseudo-data sets obtained from Monte Carlo simulations, for the cascade sample, produces the sensitivity and discovery fluxes shown in figure 3 for three spectral indices ( $\Gamma=2.0,2.4$, and 2.8) out of all the possible combinations. The energy ranges for the sensitivity and discovery fluxes reported in figure are computed by taking the region in which 


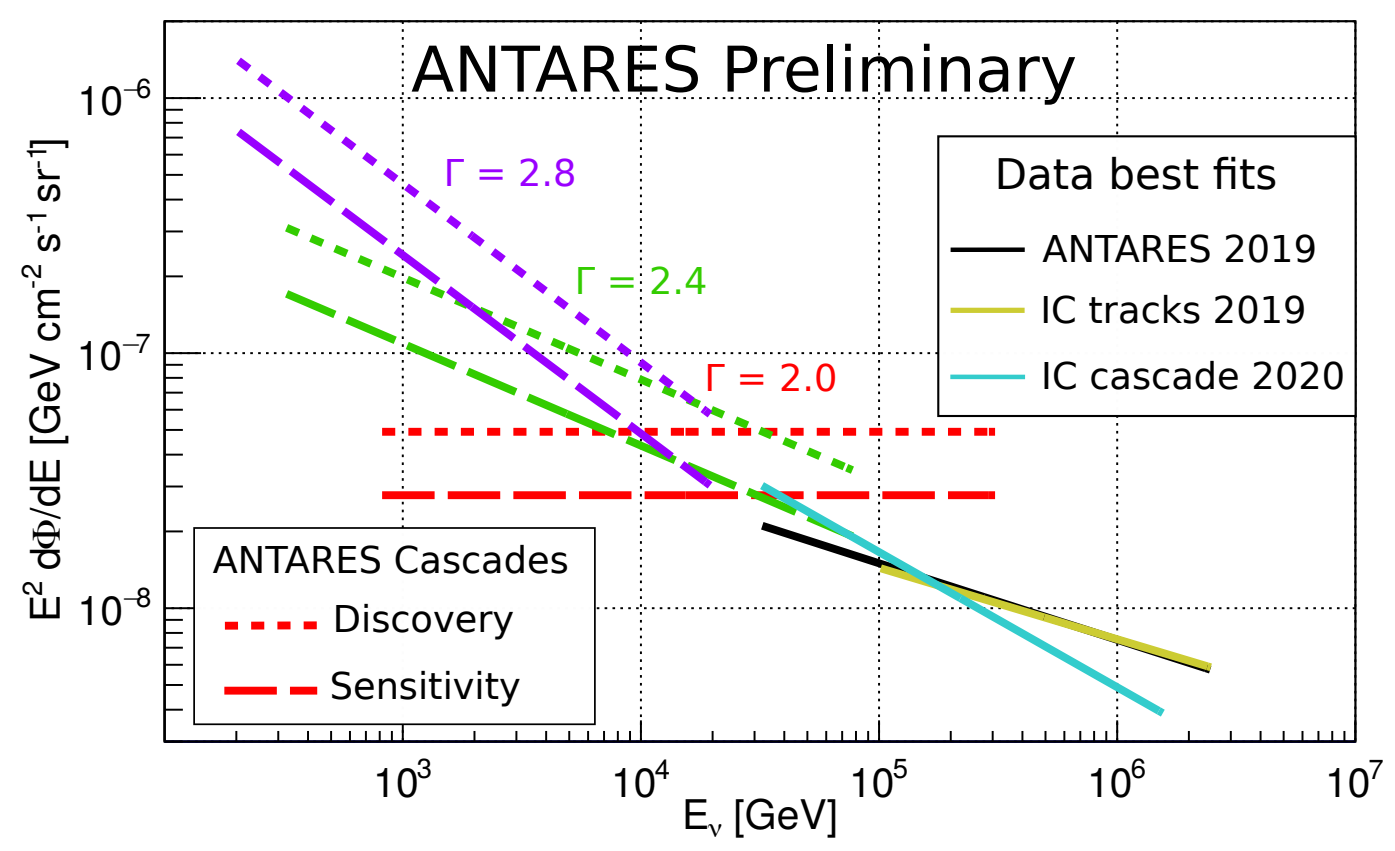

Figure 3: Cascade analysis sensitivity (dashed) and discovery potential (dotted) for three spectral indices (2.0 - red, 2.4 - green, 2.8 - violet) of this analysis compared to the best fit cosmic fluxes (solid lines) from ANTARES previous search [9] (black), IceCube tracks [2] (olive green) and IceCube cascade analyses [3] (turquoise).

the central $90 \%$ of signal events is expected from the Monte Carlo. Since the new cascade sample is more sensitive to low energies with respect to any previous search, this new analysis expands the sensitivity energy range down to the $\mathrm{TeV}$ range for the first time in searches for diffuse fluxes of cosmic neutrinos. Indeed, while previous results from IceCube and ANTARES itself were mostly sensitive around $100 \mathrm{TeV}$, the new event selection developed in ANTARES would add many more events from the low-energy tail of the cosmic distribution if an unbroken power-law is assumed also in this region. Thus, this new analysis would allow testing for the first time if also a break in the spectrum is present below $10 \mathrm{TeV}$.

An understanding of the low-energy regime of the diffuse cosmic flux is crucial for two main reasons. Energy spectra that are too soft would overshoot the $\gamma$-neutrino correlation when considering the diffuse extra-galactic photons detected by Fermi-LAT [18] and such a constrain would improve our knowledge in this energy range. Secondly, softer spectra might be tracing more local features of the overall flux, such as cosmic ray interactions within the Local Bubble or other possible propagation features that might pop-up in the diffuse cosmic spectrum as a bump in the 10 $\mathrm{TeV}$ region [17].

This search has been so far developed on Monte Carlo simulations. The next step is foreseen to happen soon and will involve analysing the real data-set collected by ANTARES between 2007 and 2020. The results above show that even though the possible observation of a cosmic neutrino component in the diffuse neutrino flux observed by ANTARES would not be very significant, it would still provide interesting insights in the study of this cosmic flux that is still largely unknown. 


\section{References}

[1] R. Abbasi et al., arXiv:2011.03545

[2] J. Stettner, PoS(ICRC2019)1017

[3] M.G. Aartsen et al., Phys. Rev. Lett. 125, 121104 (2020)

[4] M. G. Aartsen et al., Astrophys. Jour. 809, 98 (2015)

[5] A. Palladino and W. Winter, Astronomy \& Astrophysics 615, 168 (2018)

[6] M.G. Aartsen et al., Science 361, 147 (2018)

[7] A. Albert et al., Astrophys. Journ. Lett. 868, 20 (2018)

[8] A. Albert et al., Astrophys. Journ. Lett. 853, 7 (2018)

[9] L.A. Fusco and F. Versari, PoS(ICRC2019)891

[10] A. Albert et al., Phys. Lett. B 816, 136228 (2021)

[11] S. Adrian-Martinez et al., Astrophys. Journ. 760, 53 (2012)

[12] A. Albert et al., Astronom. Journ. 154, 275 (2017)

[13] G.C. Hill and K. Rawlins, Astropart. Phys. 19, 393 (2003)

[14] M. Honda et al., Physics Review D 75, 043006 (2007)

[15] R. Enberg, M.H. Reno and I. Sarcevic, Phys. Rev. D 78, 043005 (2008)

[16] G. Carminati et al., Comput. Phys. Commun. 179, 915 (2008)

[17] K.J. Andersen, M. Kachelriess and D.V. Semikoz, Astrophys. Journ. Lett. 861, 19 (2018)

[18] M. Ahlers and F. Halzen, Rept. Prog. Phys. 78 12, 126901 (2015) 


\section{Full Authors List: ANTARES Collaboration}

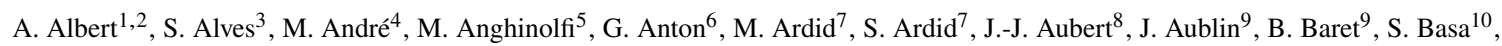

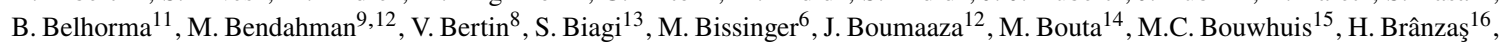
R. Bruijn ${ }^{15,17}$, J. Brunner ${ }^{8}$, J. Busto ${ }^{8}$, B. Caiff ${ }^{5}$, A. Capone ${ }^{18,19}$, L. Caramete ${ }^{16}$, J. Carr ${ }^{8}$, V. Carretero ${ }^{3}$, S. Celli ${ }^{18,19}$, M. Chabab ${ }^{20}$, T. N. Chau ${ }^{9}$, R. Cherkaoui El Moursli ${ }^{12}$, T. Chiarusi ${ }^{21}$, M. Circella $^{22}$, A. Coleiro ${ }^{9}$, M. Colomer-Molla ${ }^{9,3}$, R. Coniglione $^{13}$, P. Coyle $^{8}$, A. Creusot $^{9}$, A. F. Díaz ${ }^{23}$, G. de Wasseige ${ }^{9}$, A. Deschamps ${ }^{24}$, C. Distefano ${ }^{13}$, I. Di Palma ${ }^{18,19}$, A. Domi ${ }^{15,17}$, C. Donzaud ${ }^{9,25}$, D. Dornic ${ }^{8}$, D. Drouhin ${ }^{1,2}$, T. Eberl ${ }^{6}$, T. van Eeden ${ }^{15}$, D. van Eijk ${ }^{15}$, N. El Khayati ${ }^{12}$, A. Enzenhöfer ${ }^{8}$, P. Fermani ${ }^{18,19}$, G. Ferrara $^{13}$, F. Filippini ${ }^{21,26}$, L.A. Fusco ${ }^{8}$, Y. Gatelet ${ }^{9}$, P. Gay ${ }^{27,9}$, H. Glotin $^{28}$, R. Gozzini ${ }^{3}$, R. Gracia Ruiz ${ }^{15}$, K. Graf ${ }^{6}$, C. Guidi ${ }^{5,29}$, S. Hallmann ${ }^{6}$, H. van Haren ${ }^{30}$, A.J. Heijboer ${ }^{15}$, Y. Hello ${ }^{24}$, J.J. Hernández-Rey ${ }^{3}$, J. Höß1 ${ }^{6}$, J. Hofestädt ${ }^{6}$, F. Huang ${ }^{8}$, G. Illuminati ${ }^{9}, 21,26$, C.W James ${ }^{31}$, B. Jisse-Jung ${ }^{15}$, M. de Jong ${ }^{15,32}$, P. de Jong ${ }^{15}$, M. Kadler ${ }^{33}$, O. Kalekin ${ }^{6}$, U. Katz ${ }^{6}$, N.R. Khan-Chowdhury ${ }^{3}$, A. Kouchner ${ }^{9}$, I. Kreykenbohm ${ }^{34}$, V. Kulikovskiy ${ }^{5,36}$, R. Lahmann ${ }^{6}$, R. Le Breton ${ }^{9}$, D. Lefèvre ${ }^{35}$, E. Leonora ${ }^{36}$, G. Levi ${ }^{21,26}$, M. Lincetto ${ }^{8}$, D. Lopez-Coto ${ }^{37}$, S. Loucatos ${ }^{38,9}$, L. Maderer ${ }^{9}$, J. Manczak ${ }^{3}$, M. Marcelin ${ }^{10}$, A. Margiotta ${ }^{21,26}$, A. Marinelli ${ }^{39}$, J.A. Martínez-Mora ${ }^{7}$,

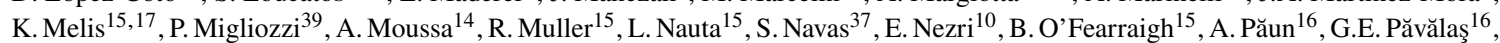
C. Pellegrino ${ }^{21,40,41}$, M. Perrin-Terrin ${ }^{8}$, V. Pestel ${ }^{15}$, P. Piattelli ${ }^{13}$, C. Pieterse ${ }^{3}$, C. Poirè ${ }^{7}$, V. Popa ${ }^{16}$, T. Pradier ${ }^{1}$, N. Randazzo ${ }^{36}$, S. Reck ${ }^{6}$, G. Riccobene ${ }^{13}$, A. Romanov ${ }^{5,29}$, A. Sánchez-Losa ${ }^{3,22}$, F. Salesa Greus ${ }^{3}$, D. F. E. Samtleben ${ }^{15,32}$, M. Sanguineti ${ }^{5,29}$, P. Sapienza ${ }^{13}$, J. Schnabel ${ }^{6}$, J. Schumann ${ }^{6}$, F. Schüssler ${ }^{38}$, M. Spurio ${ }^{21,26}$, Th. Stolarczyk ${ }^{38}$, M. Taiuti ${ }^{5,29}$, Y. Tayalati ${ }^{12}$, S.J. Tingay ${ }^{31}$, B. Vallage ${ }^{38,9}$, V. Van Elewyck ${ }^{9,41}$, F. Versari ${ }^{21,26,9}$, S. Viola ${ }^{13}$, D. Vivolo ${ }^{39,43}$, J. Wilms ${ }^{34}$, S. Zavatarelli ${ }^{5}$, A. Zegarelli ${ }^{18,19}$, J.D. Zornoza ${ }^{3}$, and J. Zúñiga ${ }^{3}$

${ }^{1}$ Université de Strasbourg, CNRS, IPHC UMR 7178, F-67000 Strasbourg, France. ${ }^{2}$ Université de Haute Alsace, F-68100 Mulhouse, France. ${ }^{3}$ IFIC - Instituto de Física Corpuscular (CSIC - Universitat de València) c/ Catedrático José Beltrán, 2 E-46980 Paterna, Valencia, Spain. ${ }^{4}$ Technical University of Catalonia, Laboratory of Applied Bioacoustics, Rambla Exposició, 08800 Vilanova i la Geltrú, Barcelona, Spain. ${ }^{5}$ INFN - Sezione di Genova, Via Dodecaneso 33, 16146 Genova, Italy. ${ }^{6}$ Friedrich-Alexander-Universität Erlangen-Nürnberg, Erlangen Centre for Astroparticle Physics, Erwin-Rommel-Str. 1, 91058 Erlangen, Germany. ${ }^{7}$ Institut d'Investigació per a la Gestió Integrada de les Zones Costaneres (IGIC) - Universitat Politècnica de València. C/ Paranimf 1, 46730 Gandia, Spain. ${ }^{8}$ Aix Marseille Univ, CNRS/IN2P3, CPPM, Marseille, France. ${ }^{9}$ Université de Paris, CNRS, Astroparticule et Cosmologie, F-75013 Paris, France. ${ }^{10}$ Aix Marseille Univ, CNRS, CNES, LAM, Marseille, France. ${ }^{11}$ National Center for Energy Sciences and Nuclear Techniques, B.P.1382, R. P.10001 Rabat, Morocco. ${ }^{12}$ University Mohammed V in Rabat, Faculty of Sciences, 4 av. Ibn Battouta, B.P. 1014, R.P. 10000 Rabat, Morocco. ${ }^{13}$ INFN - Laboratori Nazionali del Sud (LNS), Via S. Sofia 62, 95123 Catania, Italy. ${ }^{14}$ University Mohammed I, Laboratory of Physics of Matter and Radiations, B.P.717, Oujda 6000, Morocco. ${ }^{15}$ Nikhef, Science Park, Amsterdam, The Netherlands. ${ }^{16}$ Institute of Space Science, RO-077125 Bucharest, Măgurele, Romania. ${ }^{17}$ Universiteit van Amsterdam, Instituut voor Hoge-Energie Fysica, Science Park 105, 1098 XG Amsterdam, The Netherlands. ${ }^{18}$ INFN - Sezione di Roma, P.le Aldo Moro 2, 00185 Roma, Italy. ${ }^{19}$ Dipartimento di Fisica dell'Università La Sapienza, P.le Aldo Moro 2, 00185 Roma, Italy. ${ }^{20}$ LPHEA, Faculty of Science - Semlali, Cadi Ayyad University, P.O.B. 2390, Marrakech, Morocco. ${ }^{21}$ INFN - Sezione di Bologna, Viale Berti-Pichat 6/2, 40127 Bologna, Italy. ${ }^{22}$ INFN - Sezione di Bari, Via E. Orabona 4, 70126 Bari, Italy. ${ }^{23}$ Department of Computer Architecture and Technology/CITIC, University of Granada, 18071 Granada, Spain. ${ }^{24}$ Géoazur, UCA, CNRS, IRD, Observatoire de la Côte d'Azur, Sophia Antipolis, France. ${ }^{25}$ Université Paris-Sud, 91405 Orsay Cedex, France. ${ }^{26}$ Dipartimento di Fisica e Astronomia dell'Università, Viale Berti Pichat 6/2, 40127 Bologna, Italy. ${ }^{27}$ Laboratoire de Physique Corpusculaire, Clermont Université, Université Blaise Pascal, CNRS/IN2P3, BP 10448, F-63000 Clermont-Ferrand, France. ${ }^{28}$ LIS, UMR Université de Toulon, Aix Marseille Université, CNRS, 83041 Toulon, France. ${ }^{29}$ Dipartimento di Fisica dell'Università, Via Dodecaneso 33, 16146 Genova, Italy. ${ }^{30}$ Royal Netherlands Institute for Sea Research (NIOZ), Landsdiep 4, $1797 \mathrm{SZ}$ 't Horntje (Texel), the Netherlands. ${ }^{31}$ International Centre for Radio Astronomy Research - Curtin University, Bentley, WA 6102, Australia. ${ }^{32}$ Huygens-Kamerlingh Onnes Laboratorium, Universiteit Leiden, The Netherlands. ${ }^{33}$ Institut für Theoretische Physik und Astrophysik, Universität Würzburg, Emil-Fischer Str. 31, 97074 Würzburg, Germany. ${ }^{34}$ Dr. Remeis-Sternwarte and ECAP, Friedrich-Alexander-Universität Erlangen-Nürnberg, Sternwartstr. 7, 96049 Bamberg, Germany. ${ }^{35}$ Mediterranean Institute of Oceanography (MIO), Aix-Marseille University, 13288, Marseille, Cedex 9, France; Université du Sud Toulon-Var, CNRS-INSU/IRD UM 110, 83957, La Garde Cedex, France. ${ }^{36}$ INFN - Sezione di Catania, Via S. Sofia 64, 95123 Catania, Italy. ${ }^{37}$ Dpto. de Física Teórica y del Cosmos \& C.A.F.P.E., University of Granada, 18071 Granada, Spain. ${ }^{38}$ IRFU, CEA, Université Paris-Saclay, F-91191 Gif-sur-Yvette, France. ${ }^{39}$ INFN - Sezione di Napoli, Via Cintia 80126 Napoli, Italy. ${ }^{40}$ Museo Storico della Fisica e Centro Studi e Ricerche Enrico Fermi, Piazza del Viminale 1, 00184, Roma. ${ }^{41}$ INFN - CNAF, Viale C. Berti Pichat 6/2, 40127, Bologna. ${ }^{42}$ Institut Universitaire de France, 75005 Paris, France. ${ }^{43}$ Dipartimento di Fisica dell'Università Federico II di Napoli, Via Cintia 80126, Napoli, Italy. 